BANGLADESH J CHILD HEALTH 2011; VOL 35 (3): 102-107

\title{
Epidemiological Characteristics of Human Rabies at Infectious Disease Hospital, Dhaka
}

\author{
MD. SHAHIDUL HAQUE ${ }^{1}$, TAHMINA YEASMIN², MD. MANIRUL ISLAM ${ }^{3}$
}

\begin{abstract}
Objective: To describe the epidemiological characteristics of human rabies at IDH, Dhaka in 2006. Methods: Analysis of the records of rabies cases admitted into the Infectious Disease Hospital, Dhaka in 2006 (Jan-Dec). Results: Of total 170 rabies cases, $116(68.2 \%)$ cases were admitted to IDH from Dhaka division and rest 44(31.8\%) cases came from other areas of Bangladesh. Majority (59.5\%) of the victims were within 15 years of age. Male (67.1\%) gender \& rural people $(89.4 \%)$ constituted the main bulk of the cases. $86.5 \%$ cases had WHO category III exposure. Majority cases had incubation period between 16-90 days (82.9\%). Hydrophobia which is the main pathognomonic feature of human rabies, was present in cent percent cases. Principal biting animals was dog (92.94\%). Others animals were cat(5.3\%),jackal (0.6\%), mongoose (1.2\%). Most cases $(143 / 170=84.1 \%)$ were not vaccinated. $99 \%$ cases did not take wound care. Among 27 cases who received vaccines, only 6 cases (22.2\%) received TCV inadequately and remaining 21(77.8\%) received NTV. None of 170 cases were treated with RIG. Conclusion: Rabies is a major health problem in Bangladesh. The data indicates that the epidemiological characteristics of the disease are not much different from other studies and have not changed much over the decades. Hence, there are need to educate the community and health workers about the importance of immediate and adequate post-exposure treatment, to start effective control programme for dog and to make availability of TCV as well as RIG.
\end{abstract}

Key words: Rabies, Anti rabies vaccines, Hydrophobia

\section{Introduction}

Rabies is a zoonotic disease transmitted to human by the bite or scratch or licks (on broken skin or mucous membrane) of an infected animal, most often a dog. If left untreated, the bite of a rabid animal invariably leads to dramatically painful death. Although there is no effective treatment once symptoms are established, but it can be prevented by effective measures like immediate wound washing, wound infiltration with rabies immunoglobulin and a course of vaccination using $\mathrm{WHO}$ recommendation.

\section{Cell culture vaccine.}

Human mortality from endemic canine rabies is estimated by $\mathrm{WHO}$ to be around 55000 death annually with over 31000 death in Asia alone. ${ }^{1}$ An estimated 6 million people undergo post exposure treatment (PET) of rabies annually worldwide. ${ }^{2}$ In the

1. Assistant Professor, Faculty of Paediatrics, Institute of Child and Mother Health, Matuail, Dhaka. Ex- Junior Consultant(Paed) IDH, Mohakhali, Dhaka.

2. Department of Physiology, Dhaka Medical College. Dhaka.

3. Training Physician, ICDDRB. Dhaka.

Correspondence: Dr. Md. Shahidul Haque
United states approximately 40,000 people receive PET annually while there has been an average of three fatal cases of human rabies cases per year since $1980 .^{3}$

Rabies is an important health problem in Bangladesh. Bangladesh is an endemic zone of rabies distributed uniformly throughout the country, but the exact magnitude of the disease is not known. Experts estimate about 2000 rabies death occur every year after getting scattered record from different Infectious Diseases Hospitals (IDH) in Bangladesh. There are six IDH in Bangladesh. But the IDH in Dhaka is the largest one and majority of rabies patients come to this hospital from throughout the country. About 1 lac patients of animal bite received post-exposure prophylaxis annually. The majority patient received nerve tissue vaccine (NTV) of very low price. This vaccine is produced in Bangladesh \& is available. The tissue culture vaccine (TCV) is not produced in Bangladesh but is imported from foreign countries. Two costly tissue culture vaccines(Rabipur \& Verorab) are available in Bangladesh. The use of TCV is 
gradually increasing day by day by those who can afford this costly vaccine. The use of rabies immunoglobulin (RIG) is very limited, because of high price and inadequate supply. RIG is also an imported item.

In Infectious Diseases Hospital at Dhaka, about 150200 rabies patients are admitted per year. According to hospital records, the annual number of cases of rabies at the IDH, Dhaka has not been changed significantly over the last 10 years.

This descriptive type of study was done retrospectively to see the epidemiological characteristics of the rabies victims admitted in IDH, Dhaka, in 2006.

\section{Materials \& Methods}

Infectious Diseases Hospital (IDH), Dhaka is the only hospital of this kind in Dhaka and it is common practice for the physician of this area to refer rabies cases to this hospital. Rabies cases are also referred from other area of whole Bangladesh. Hospital records of 170 clinically diagnosed cases of rabies (having hydrophobia) admitted to the IDH, Dhaka in 2006 were analyzed retrospectively. Most of them also have aerophobia. Clinical case sheet had data on age, sex, date of admission, place of bite, type \& name of animal, duration between bite \& development of symptom, type of symptoms, name of vaccine given, duration between bite \& administration of vaccine, fate of animal, rabies immunoglobulin taken or not. Cases were categorized for the degree of exposure on the basis of criteria recommended by $\mathrm{WHO}^{4}$

Some of the clinical \& epidemiological details were not available from the case records of the hospital. Analysis contain only those information that were available.

The records were analyzed by computer using SPSS version 13.0 soft ware.

\section{Results}

In 2006, 170 cases of clinically diagnosed rabies were admitted into IDH, Dhaka. Of these 116(68.2\%) cases were from Dhaka division. The remaining cases came from Chittagong 34(20.1\%), Sylhet 2(1.2\%), Barishal 5(2.9\%), Rajshahi 9(5.3\%) and Khulna 4(2.4\%) division (Table-I). Most cases came from the rural areas 152(89.4\%). The cases came to IDH throughout the year (12 to 25 cases per month) and no particular seasonality could be identified.
Table-I

Division \& area wise distribution of human rabies cases

\begin{tabular}{lccc}
\hline Division & Urban & Rural & Total \\
& $\mathrm{N}(\%)$ & $\mathrm{N}(\%)$ & $\mathrm{N}(\%)$ \\
\hline Dhaka & $16(13.8)$ & $100(86.2)$ & $116(68.2)$ \\
Chittagong & $2(5.9)$ & $32(94.1)$ & $34(20.1)$ \\
Sylhet & 0 & 2 & $2(1.2)$ \\
Barishal & 0 & 5 & $5(2.9)$ \\
Rajshahi & 0 & 9 & $9(5.3)$ \\
Khulna & 0 & 4 & $4(2.4)$ \\
\hline Total & $18(10.6)$ & $152(89.4)$ & $170(100)$ \\
\hline
\end{tabular}

Table-II describes the cases by age and sex. About $101(59.5 \%)$ cases were children under 15 years of age, of which $75(44.1 \%)$ were in the age group of 5 to 14 years. Male contribute to about $67.1 \%(114 / 170)$ of cases in all age groups. About $92.9 \%(158 / 170)$ of cases gave the history of dog bite, pet animals were involved in only $12(7.1 \%)$ cases. The other animals involved were cat $9(5.3 \%)$, Jackal $1(0.6 \%)$, Mongoose $2(1.2 \%)$. Stray animal and rabid animal contributed $131(77.1 \%) \& 25(15.9 \%)$ respectively, of which dog was the main animal (Table-III). None of the pet animal was vaccinated. Vaccination status of stray animals was not known.

Table-II

Age and Gender wise distribution of rabies cases

\begin{tabular}{cc}
\hline Age group & Total \\
\hline years & $\mathrm{N}(\%)$ \\
$0-4$ & $26(15.4)$ \\
$5-14$ & $75(44.1)$ \\
$15-34$ & $33(19.4)$ \\
$35-54$ & $27(15.8)$ \\
$>55$ & $9(5.2)$ \\
Gender & \\
Male & $114(67.1)$ \\
Female & $56(32.9)$ \\
\hline Total & $170(100)$ \\
\hline
\end{tabular}


Table-III

Type and name of animal involved

\begin{tabular}{lcc}
\hline Type & Number & Percentage (\%) \\
\hline Stray & 131 & 77.1 \\
Rabid & 27 & 15.9 \\
Pet & 12 & 7.1 \\
Total & 170 & 100 \\
Species & & \\
Dog & 158 & 92.9 \\
Cat & 9 & 5.3 \\
Jackal & 1 & 0.6 \\
Mongoose & 2 & 1.2 \\
\hline Total & 170 & 100 \\
\hline
\end{tabular}

Incubation period was calculated as a difference of the date of animal bite and date of onset of symptoms (Table-IV). Thirty four point seven percent (34.7\%) persons developed the disease between 16-30 days. Ninety nine point four (99.4\%) developed symptoms in less than a year. In the present study only $0.6 \%$ people had incubation period more than 1 year. The mean \& median incubation periods were $78.28 \& 60$ days respectively. Hydrophobia, which is the predominant pathognomonic clinical feature of the disease, was present in $100 \%$ cases. Along with other clinical features hydro-aerophobia was present in $85.9 \%$ cases. No case was presented with only aerophobia. But $11.8 \%$ cases were presented with only hydrophobia (Table-V).

Table-IV

Incubation period of human rabies cases

\begin{tabular}{lccc}
\hline $\begin{array}{l}\text { Period } \\
\text { (Days) }\end{array}$ & Number & Percentage & $\begin{array}{c}\text { Cumulative } \\
\text { Percentage }\end{array}$ \\
\hline $16-30$ & 59 & 34.7 & 34.7 \\
$31-60$ & 46 & 27.1 & 61.8 \\
$61-90$ & 36 & 21.2 & 82.9 \\
$91-180$ & 17 & 10 & 92.9 \\
$181-365$ & 11 & 6.5 & 99.4 \\
$366-730$ & 1 & 0.6 & 100 \\
\hline
\end{tabular}

Minimum- 18 days, Maximum- 390 days, Mean- 78.28

days

Median- 60 days
Table-V

Clinical features of human rabies cases

\begin{tabular}{lcc}
\hline Presenting features & Number & Percentage \\
\hline Hydrophobia & 20 & 11.8 \\
$\begin{array}{l}\text { Hydro-aerophobia } \\
\text { Hydro-aerophobia with }\end{array}$ & 146 & 85.9 \\
$\begin{array}{l}\text { Restlessness } \\
\text { Hydro-aerophobia with }\end{array}$ & 1 & 0.6 \\
Restlessness \& salivation & & 1.8 \\
\hline Total & 170 & 100 \\
\hline
\end{tabular}

Of the total 170 persons bitten, 23(13.5\%) had category II (Abrasion, minor scratch without bleeding, licks on broken skin) and $147(86.5 \%)$ had category III ( single or multiple transdermal bites or scratches or licks on mucous membrane) exposure (Table 6.). One hundred \& forty five $(85.3 \%)$ persons had bite on limbs. Fourteen (8.2\%) \& 11 (6.5\%) cases had bite on face $\&$ or head or neck, and trunk respectively. Only 5 cases had both limb and trunk bite.

Table-VI

Category of animal bite according to WHO guideline ${ }^{4}$

\begin{tabular}{lcc}
\hline Type & Number & Percentage \\
\hline Type II & 23 & 13.5 \\
Typelll & 147 & 86.5 \\
\hline Total & 170 & 100 \\
\hline
\end{tabular}

The fate of 82 (48.2\%) biting animal was not known as victim or their relatives did not observe them. Seventeen $(10 \%)$ animals died within 10 days of bite and $47(27.6 \%)$ were killed as they had bitten other persons. Surprisingly, 24(14.1\%) biting animals were alive (Table-VII). Twenty seven (15.88\%) persons gave the history that same dog had bitten 3 to 10 persons and $14(8.23 \%)$ persons said that they were the single victim of an animal bite. However, 129(75.88\%) persons did not know whether the animal had bitten any other person also. One hundred \& forty nine $(87.64 \%)$ persons did not take or receive any wound treatment. Twelve $(7.05 \%)$ washed the wound with soap \& water and $9(5.29 \%)$ had applied antiseptics without washing the wound.

Table-VII

Fate of biting animals

\begin{tabular}{lcc}
\hline Fate & Number & Percentage \\
\hline Died & 17 & 10 \\
Killed & 47 & 27.6 \\
Alive & 24 & 14.2 \\
Not found /Run away & 82 & 48.2 \\
\hline Total & 170 & 100 \\
\hline
\end{tabular}


Table-VIII

Post- exposure anti-rabies vaccination $(n=170)$

\begin{tabular}{lcccc}
\hline Variables & $\begin{array}{c}\text { Vaccination } \\
\text { Not taken } \\
\text { Residence }\end{array}$ & $\begin{array}{c}\text { Complete } \\
\text { Vac. taken } \\
\mathrm{N}(\%)\end{array}$ & $\begin{array}{c}\text { Partial } \\
\text { Vac. taken } \\
\mathrm{N}(\%)\end{array}$ & $\mathrm{N}(\%)$ \\
\hline Dhaka & $94(81.6)$ & $14(12.1)$ & $8(6.9)$ & $116(68.2)$ \\
Outside Dhaka & $49(90.7)$ & $2(3.7)$ & $3(5.5)$ & $54(31.7)$ \\
Sex & & & & \\
Male & $91(79.8)$ & $14(12.3)$ & $9(7.9)$ & $114(67.0)$ \\
Female & $52(92.9)$ & $2(3.6)$ & $2(3.6)$ & $56(32.9)$ \\
Age group (years) & & & & \\
0-4 & $23(88.4)$ & $2(7.6)$ & $1(3.8)$ & $26(15.2)$ \\
$5-14$ & $63(84)$ & $9(12)$ & $3(4)$ & $75(44.1)$ \\
$15-34$ & $26(78.7)$ & $3(9.1)$ & $4(12.1)$ & $33(19.4)$ \\
$35-54$ & $24(88.8)$ & $1(3.7)$ & $2(7.4)$ & $27(15.8)$ \\
e"55 & $7(77.7)$ & $1(11.1)$ & $1(11.1)$ & $9(5.2)$ \\
\hline Total & $143(84.1)$ & $16(9.4)$ & $11(6.4)$ & $170(100)$ \\
\hline
\end{tabular}

** NTV-21cases, Verorab- 2 cases, Rabipur- 4 cases.

One hundred \& forty three (84.1\%) persons did not receive a single dose of vaccination. Out of remaining $27(15.8 \%)$ persons, $16(9.4 \%)$ and $11(6.4 \%)$ persons received complete and partial anti rabies vaccination respectively (Table-VIII). Twenty one (12.3\%) received nerve tissue vaccine (NTV) and only 6(3.5\%) received tissue culture vaccine (TCV) (Rabipur-4, Verorab -2). None received anti rabies serum or immunoglobulin.

Only $7(4.1 \%)$ people died in the hospital whereas $163(96.8 \%)$ left the hospital in critical condition against medical advice after learning about the fatal outcome.

\section{Discussion}

Exact data on magnitude of human rabies in Bangladesh is not available. However, scattered hospital records indicate that about 2000 people die from rabies each year in Bangladesh. Major portion of rabies patients comes to IDH, Dhaka from the whole country every year. Rabies is almost cent percent fatal disease, but easily preventable disease if animal bites are appropriately \& timely managed. ${ }^{5}$ Despite trial with various antivirus agents, there is no therapy of proven value once the disease is manifested. ${ }^{6}$ Appropriate care of wound, adequate post-exposure treatment (PET) with modern TCV with RIG ( when indicated ) according to WHO guideline can prevent the disease amongst most of the persons exposed to rabid animals. ${ }^{7-8}$ In this present study, cases occurred in all age groups, but majority of cases $(101 / 170=59.5 \%)$ were under 15 years of age. This age group reflects that children were the principal victim, because of their inherent fondness to animals and their short statures get bitten close to head \& neck and hence to develop the disease faster if not timely \& appropriately treated. Occurrence of $67.1 \%$ of the cases in males clearly indicates that it is a exposure related disease and men in our society move out of the houses more than female for occupation. Similar age and gender has been reported by other authors $^{9-12}$.

The chance of developing the diseases were found to be greatest $(61.8 \%)$ between $30-60$ days of postexposure. Except for $0.6 \%$ cases with an incubation period of more than one year, the remaining (39.2\%) cases developed the disease within 12 month of exposure. Similar observations regarding the duration of incubation period have also been made by other authors. ${ }^{9-13}$ There are many misconceptions associated with animal bite management which deprive the patients of getting the right post-exposure treatment (PET) in Bangladesh. Poverty, ignorance and faith in indigenous medicines are more common in rural people and it is surprising that cases belonged to the rural areas $(152=89.4 \%)$ contributed to the main bulk of the cases in this study. Moreover, one 
can seldom see such animal in urban area as urbanization has isolated people from the natural interaction in our country. In this present study, none of the patients had received appropriate post-exposure treatment timely. About $99 \%$ cases did not received wound care. From the present study and earlier studies it is revealed that the people are either not aware of its importance or had no confidence of its usefulness as an anti-rabies measure. ${ }^{9-10}$

Although 147 (86.5\%) cases had category III exposure, none of the cases were given RIG. This is an essential component of rabies post-exposure treatment, especially in persons who would have short incubation period ( Category III exposure with bite on head \& neck area) because it takes at least 10 days to produce immunity following primary vaccination. RIG was not available during the study period in Bangladesh. Although at this moment limited amount of RIG is available in Dhaka (imported from India), its price is high and its unavailability in other parts of the country make it difficult to provide correct PET. Eighty four point one percent $(143 / 170)$ of cases were never administered even a single dose of antirabies vaccine. Out of 27 cases who had been administered antirabies vaccine, only 16 cases actually completed the doses, the rest 11 cases could not be completed the doses due to unavailability of vaccine and highly distant rural area. NTV of unreliable potency and high rate of associated complications are still being used as the main weapon of PET in Bangladesh and in developing countries. 9,14,15 Many persons especially children fear to take NTV as part of PET of rabies in Bangladesh. Modern tissue culture vaccine (TCV) are more effective and safer, but are expensive and can not be afforded by most of the poor people of this country. In this study only 6 cases were given TCV but none completed the course. It is necessary that efforts may be made to make available low cost TCV for all victims of bite in the entire country.

In the present study, $100 \%$ of cases presented with hydrophobia which is the most characteristic \& widely Known feature of the disease along with other symptoms. This finding is comparable to another study in India. ${ }^{11}$

Like many countries dog is the principal animal ( 158/ $170=92.9 \%$ ) responsible for transmission of the disease to human in this study as has been reported in many previous studies. ${ }^{7-9,14-15}$ So effective measure to control canine rabies are necessary to reduce human rabies in Bangladesh. Pet animals may get infected from rabid animal. Those who ignored the bite from an apparently healthy but rabid animal and did not take appropriate treatment paid the ultimate price. So pet are not always safe as far as the rabies is concerned and should be vaccinated. Pet animal transmitted the disease to human in $7.1 \%(12 / 170)$ of cases in this study which is comparable to findings in the neighboring country. ${ }^{9}$ Interestingly in this study $14.2 \%(24 / 170)$ of cases reported that the animal were still alive when they were admitted to IDH. Similar finding was found in other studies in India. ${ }^{7,11}$ So this finding suggests that one must get proper PET without observing whether the animal is rabid or non rabid. It is necessary that all animal bite in endemic areas are considered to be rabid animals \& should be given post exposure treatment as per guideline. ${ }^{8}$

\section{Conclusion}

The epidemiological characteristics of human rabies in Bangladesh found in this study are not much different from other studies. Rabies remains an important cause of mortality in the developing countries and is difficult to eliminate from a country like Bangladesh. However, effective control of canine rabies, availability of adequate modern post exposure treatment with TCV \& RIG ( when indicated) according to WHO guideline, health education, committed \& coordinated action from different government and nongovernment organization can only make a rabies free Bangladesh.

\section{Acknowledgements}

The authors are grateful and thankful to doctors and other health personals of the emergency department of the Infectious Disease Hospital, Dhaka.

\section{References:}

1. Knobel DL, Cleaveland S, Coleman PG, Fevre EM, Meltzer MI, Miranda ME, et al. Re-evaluating the burden of rabies in Africa \& Asia. Bull World Health Organ 2005; 83(5): 360-8.

2. Wilde H. Rabies 1996. International Journal of Infectious Disease 1997; 1(3): 135-42.

3. Messenger SL, Smith JS, Rupprecht CE. Emerging epidemiology of bat-associated cryptive cases of rabies in human in United States. Clin Infect Dis 2002; 356:738-47.

4. World Health Organization. WHO Recommendation of Rabies Post- exposure Treatment and the Correct Technique of 
Intradermal Immunization Against Rabies. WHO document: WHO/ EMC/ 2000; 96: 6,1997.

5. World Health Organization.WHO expert consultation on rabies. WHO Tech Rep Ser 2005; Abstract 931, p-88.

6. Jackson AC, Warrell MJ, Rapprecht CE, Ertl $\mathrm{HCJ}$, Dietzschold B,O'Reilly $M$, et al. Management of rabies in human. Cinical Infectious Diseases 2003; 36(1): 60-63.

7. Center for Disease Control and Prevention- United States, 1999: Recommendations of Advisory Committee on Immunization Practices (ACIP). MMWR Morb Mortal Wkly Rep 1999; 48(RR-1): 1-21.

8. WHO expert committee on rabies. World Health Organ Tech Rep Ser, 1992; 824: 1-84.

9. Singh J, Jain DC, Bhatia R, Ichhpujani RL, Harit AK, Panda RC, et al. Epidemiological characteristics of rabies in Delhi and surrounding areas, 1998. Indian Paediatr 2001; 38(12): 135460.

10. Lakhanpal U, Sharma RC. An epidemiological study of 177 cases of human rabies. Int J Epidemiolgy 1985;14(4): 614-17.
11. Chowdhury JR, Modak KK. Report on Rabies in West Bengal. J Indian Med Assoc 1983; 81(5\& 6): 69-74.

12. Rahman M, Salimuzzamn M, Alam MB, Rouf MA, Hossain MJ, Rahman MR. Human rabies in Bangladesh-A study of 684 cases. J Medicine 2007; 8: 3-6.

13. Chhabra M, Ichhpujani RL, Tewari KN, Lal S. Human Rabies in Delhi. Indian J Paediatr 2004; 71(3): 217-20.

14. Wilde H, Briggs DJ, Meslin FX, Hemachudha T, Sitprija V. Rabies updates for travel medicine advisors. Clinical Infectious Diseases 2003; 37(1): 96-100.

15. World Health Organization. Strategies for the control and elimination of rabies in Asia: report of an interregional consultation. Document WHO/ CSR/EPT2002.8. Geneva: World Health Organization, 2002.

16. Dutta JK. Human rabies in India: Epidemiological features, management \& current methods of prevention. Trop Doct 1999; 29(4): 196-201.

17. Parviz S, Luby S, Wilde H. Post-exposure treatment of rabies in Pakistan. Clinical Infectious Diseases 1998; 27(4): 751-6. 